\title{
Repeated intravitreal injections of antivascular endothelial growth factor in patients with neovascular age-related macular degeneration may increase the risk of ischemic optic neuropathy
}

\author{
Yu-Yen Chen ${ }^{1,2,3^{*}}$ (D), Pesus Chou ${ }^{2,3}$, Yu-Fang Huang ${ }^{1}$, Hung-Jen Chien ${ }^{1}$, Yu-Chieh Wu ${ }^{1}$, Chia-Chi Lee', \\ Li-Ying Huang ${ }^{4,5,6}$ and Hsin-Hua Chen ${ }^{2,3,7,8,9}$
}

\begin{abstract}
Background: Previous case reports have demonstrated the occurrence of ischemic optic neuropathy (ION) following intravitreal injections of antivascular endothelial growth factor (anti-VEGF). However, no previous studies have investigated the impact of injection numbers on the risk of ION. The aim of our study was to investigate whether repeated intravitreal injections of anti-VEGF would increase the risk of subsequent ION in patients with neovascular age-related macular degeneration (AMD).
\end{abstract}

Methods: A population-based, retrospective cohort study using the Taiwan National Health Insurance Research Database was conducted from 2007 to 2013. Neovascular AMD patients receiving intravitreal injections of anti-VEGF during the study period were enrolled in the study cohort. Enrollees were divided into three groups according to the categorized levels of injection number (first level: < 10 times, second level: 10-15 times, and third level: > 15 times). Kaplan-Meier curves were generated to compare the cumulative hazard of subsequent ION among the three groups. Cox regression analyses were used to estimate crude and adjusted hazard ratios (HRs) for ION development with respect to the different levels of injection numbers. The confounders included for adjustment were age, sex, and comorbidities (diabetes, hypertension, hyperlipidemia, ischemic heart disease, and glaucoma).

Results: In total, the study cohort included 77,210 patients. Of these, 26,520, 38,010, and 12,680 were in the first-, second-, and third-level groups, respectively. The Kaplan-Meier method revealed that the cumulative hazards of ION were significantly higher in those who had a higher injection number. After adjusting for confounders, the adjusted HRs for ION in the second- and third-level groups were 1.91 (95\% confidence interval [Cl], 1.32-2.76) and 2.20 (95\% $\mathrm{Cl}, 1.42-3.43)$, respectively, compared with those in the first-level group.

Conclusions: Among patients with neovascular AMD, those who receive a higher number of anti-VEGF injections have a significantly higher risk of developing ION compared with individuals who receive a lower number of injections.

Keywords: Intravitreal injection, Antivascular endothelial growth factor, Neovascular age-related macular degeneration, Ischemic optic neuropathy, Cohort study, Risk factor, National Health Insurance Research Database

\footnotetext{
* Correspondence: yuyenchen.phd@gmail.com

${ }^{1}$ Department of Ophthalmology, Taichung Veterans General Hospital, 1650

Taiwan Boulevard Sect. 4, Taichung 40705, Taiwan

${ }^{2} \mathrm{~S} c h o o l$ of Medicine, National Yang-Ming University, Taipei, Taiwan

Full list of author information is available at the end of the article
}

(c) The Author(s). 2019 Open Access This article is distributed under the terms of the Creative Commons Attribution 4.0 International License (http://creativecommons.org/licenses/by/4.0/), which permits unrestricted use, distribution, and reproduction in any medium, provided you give appropriate credit to the original author(s) and the source, provide a link to the Creative Commons license, and indicate if changes were made. The Creative Commons Public Domain Dedication waiver (http://creativecommons.org/publicdomain/zero/1.0/) applies to the data made available in this article, unless otherwise stated. 


\section{Background}

Neovascular age-related macular degeneration (AMD) is characterized by the proliferation of abnormal blood vessels (neovasculature) from the choroid and can cause macular edema and severe vision loss. Intravitreal injection of steroids has the effect of decreasing macular edema [1-6]. In addition, the intravitreal injection of antivascular endothelial growth factor (anti-VEGF) can inhibits vascular endothelial growth factor (VEGF) and leads to marked decreases in neovascularization of both the choroid and retina [7-10]. Thus, intravitreal injection of anti-VEGF has been widely performed to treat neovascular AMD.

Ischemic optic neuropathy (ION), resulting from an insufficient blood supply, is the most common type of acute optic neuropathy in older patients $[11,12]$. ION is classified as anterior or posterior ION according to the affected segment of the optic nerve. Both types are further categorized into arteritic (related to vasculitis), and nonarteritic (not related to vasculitis). Of these, nonarteritic anterior ION (NAION) is the most common type. The risk factors for NAION include a crowded optic disk, diabetes, hypertension, hyperlipidemia, and ischemic heart disease [13-17]. Diminished ocular blood perfusion due to elevated intraocular pressure (IOP) or glaucoma can also lead to the occurrence of ION [1823].

Although intravitreal injection of anti-VEGF is effective for treating neovascular AMD, it may have some possible complications such as endophthalmitis, intraocular inflammation, IOP elevation, and ocular hemorrhage [24]. In addition, one rare ocular adverse effect is ION [25-27]. The underlying pathogenesis of the association between anti-VEGF and the risk of subsequent ION is still not fully known. One potential mechanism may be transient IOP elevation after intravitreal injection of anti-VEGF. Another possible explanation may be due to the properties of anti-VEGF. Anti-VEGF not only inhibits the formation of the neovasulature from the choroid, but also might result in inadvertent regression of the collateral vessels of the optic nerve head. Thus, increasing the number of injections may increase the risk of developing ION. To the best of our knowledge, no previous studies have investigated the impact of injection numbers on the risk of ION. The lack of evidence-based studies results from the rare occurrence of ION. In addition, systemic diseases such as diabetes, hypertension, hyperlipidemia, ischemic heart disease, and glaucoma, may confound the association between anti-VEGF and ION, and thus must be adjusted.

We conducted this study based on the Taiwan National Health Insurance Research Database (NHIRD) to elucidate the association between the intravitreal injection of anti-VEGF and subsequent ION. Our hypothesis is that a higher number of intravitreal injections of antiVEGF will increase the risk of subsequent ION among neovascular AMD patients. We used the whole population database and therefore had large numbers of patients as well as a high level of statistical power. The completeness of the database ensures that the diagnoses of each patient are available, and the diagnoses were made according to the generally accepted International Classification of Diagnoses, Ninth Revision, Clinical Modification (ICD-9-CM) Codes.

\section{Methods}

\section{Data source}

The NHIRD was derived from the Taiwan National Health Insurance Program, which covers more than 99\% of Taiwan's 23 million residents. The NHIRD contains all the original claims data from the program and is released annually by the National Health Research Institute in an electronically encrypted form. The NHIRD consists of diagnoses, medical prescriptions, surgical procedures, and insurance registries and was released for scientific research after personal identification numbers were encrypted. This study was approved by the institutional review board of National Yang-Ming University Hospital (2015A018).

\section{Study subjects}

We conducted a retrospective cohort study from January 1, 2007, to December 31, 2013. First, we selected patients from the NHIRD who were diagnosed with neovascular AMD (ICD-9-CM code 362.52) during the study period, which required confirmation by fundoscopy, fluorescein angiography (FA), and/or optical coherence tomography (OCT). In cases of neovascular AMD cases, FA can detect the presence of lesion and determine the size and location of the choroidal neovasculature. OCT can define the cross-sectional architecture of the retina and can reveal the presence of subretinal and intraretinal fluid. Patients diagnosed with neovascular AMD before the end of 2006 were excluded to ensure that all enrolled patients had new-onset neovascular AMD. If patients were found to have an obvious choroidal neovasculature and fluid in fundoscopy/FA/ OCT, they were treated with intravitreal anti-VEGF. Those who received intravitreal anti-VEGF injections for the treatment of neovascular AMD were included in the study cohort. During the follow-up period, fundoscopy, FA, and OCT were performed regularly. If the neovasculature and fluid resolved and if no relapse occurred, the treatment with injections was ended. According to the number of anti-VEGF injections, patients in the study cohort were further divided into three groups (first level: < 10 times, second level: 10-15 times, and third level: > 15 times). 


\section{Outcome variable}

Patients in the study cohort were followed to identify the onset of ION (ICD-9-CM codes 377.41). Those with ION before the injection of anti-VEGF were excluded to ensure that all cases were new-onset ION.

\section{Demographic variables and comorbidities}

Demographic variables such as age and sex were extracted from the database. Age was categorized into three levels: the first ( $<60$ years), the second (60-75 years), and the third ( $\geq 75$ years) levels. Risk factors for ION such as diabetes (ICD-9-CM Codes 250), hypertension (ICD-9-CM Codes 401-405), hyperlipidemia (ICD9-CM Codes 272), ischemic heart disease (ICD-9-CM Codes 410-414), and glaucoma (ICD-9-CM Codes 365) may confound the relationship between anti-VEGF and ION. Therefore, these comorbidities were identified in the medical records and included as covariates in our analyses.

\section{Statistical analysis}

The Characteristics of the study cohort are presented according to age, sex, and comorbidities (e.g., diabetes, hypertension, hyperlipidemia, ischemic heart disease, and glaucoma). Group differences (first level: $<10$ times, second level: 10-15 times, and third level: > 15 times) in these variables were analyzed by ANOVA tests (for continuous variables) and chi-square tests (for categorical variables). Then, the study cohort was followed until the occurrence of ION, dropout from the database, or the end of 2013, whichever came first. Survival analysis using the Kaplan-Meier method with the log-rank test was applied to describe and compare the cumulative hazard curves of ION according to the different levels of injection number.

Subsequently, all these variables (age, sex, number of intravitreal anti-VEGF injections, and comorbidities) were included in the Cox regression analyses. Unadjusted hazard ratios (HRs) for ION were computed according to each variable in the univariate analyses. Then, adjusted HRs for ION were derived from the multivariate analyses. Comorbidities were regarded as timedependent variables. All statistical operations were performed using the SAS statistical package, version 9.2 (SAS Institute, Cary, NC, USA).

\section{Results}

\section{Demographic and clinical characteristics of the study} sample

In total, 77,210 patients were enrolled in the study cohort. Of them, 26,520 were in the first-level group, 38, 010 were in the second-level group, and 12,680 were in the third-level group. Table 1 presents the demographic and clinical characteristics of the patients. The mean age in the study cohort was 67.4 years. The male to female ratio was 1.7:1. The prevalence of comorbidities was $35.6 \%$ for diabetes, $64.6 \%$ for hypertension, $44.3 \%$ for hyperlipidemia, and $29.8 \%$ for ischemic heart disease. Additionally, $16.5 \%$ of patients in the study cohort had glaucoma. The mean number of injections among the study cohort was 12.0 , with a standard deviation (SD) of 2.9. Almost $50 \%$ of the patients had injection numbers in the range of 10 to 15 . Significant differences were found across the three groups (first level: $<10$ times, second level: 10-15 times, and third level: $>15$ times) in age, sex, and comorbidities. The follow-up period in the study cohort was $3.50 \pm 1.86$ (mean $\pm \mathrm{SD}$ ) years, and did not differ significantly among the three groups. Of the 77,210 patients, $180(0.23 \%)$ had a subsequent occurrence of ION: $40(0.15 \%)$ in the first-level group, 100 $(0.26 \%)$ in the second-level group, and $40(0.32 \%)$ in the third-level group.

\section{Kaplan-Meier curves and log-rank test}

Figure 1 illustrates the Kaplan-Meier curves of ION corresponding to each level of injection number. According to the log-rank test, the difference in cumulative hazards was significant $(p<0.01)$.

\section{ION risk}

Table 2 displays the HRs for ION with regard to age, sex, injection number, and comorbidities. In the univariate Cox regression analysis, the second-level (10-15 times) and the third-level ( $\geq 15$ times) groups yielded unadjusted HRs for ION of 1.75 (95\% confidence interval $[\mathrm{CI}], 1.21-2.53)$ and 1.99 (95\% CI, 1.28-3.08), respectively, compared with the first-level $(<10$ times) group. The adjusted HRs obtained by comparing the second- and third level groups with the first-level group of were 1.91 (95\% CI, 1.32-2.76) and 2.20 (95\% CI, 1.42-3.43), respectively. Diabetes significantly increased the risk for ION in both the univariate and multivariate analyses. However, age, sex, hypertension, hyperlipidemia, and ischemic heart disease were not significant risk factors for ION in either the univariate or multivariate analyzes. Glaucoma increased the risk for ION in the univariate analysis, but the statistical significance was only marginal (unadjusted $\mathrm{HR}=1.43 ; 95 \% \mathrm{CI}, 1.01$ 2.03), and there was no significant difference in the multivariate analysis (adjusted $\mathrm{HR}=1.35$; 95\% CI, $0.95-$ 1.92).

\section{Discussion}

This study is the first to reveal the increased risk of ION after repeated injections of anti-VEGF among neovascular AMD patients. In our population-based study, utilizing Taiwan's NHIRD with a long (7-year) study period, we found that among patients with neovascular AMD, 
Table 1 Characteristics of the study cohort

\begin{tabular}{|c|c|c|c|c|c|}
\hline Variable & Study cohort $(n=77,210)$ & $\begin{array}{l}\text { First level } \\
(n=26,520)\end{array}$ & $\begin{array}{l}\text { Second level } \\
(n=38,010)\end{array}$ & $\begin{array}{l}\text { Third level } \\
(n=12,680)\end{array}$ & $p$-value \\
\hline Age, years & $67.4 \pm 12.2$ & $65.3 \pm 13.0$ & $68.1 \pm 12.0$ & $69.5 \pm 10.5$ & $<0.0001$ \\
\hline Age group, categorical & & & & & $<0.0001$ \\
\hline$<60$ & $20,020(25.9)$ & 8840 (33.3) & $8940(23.5)$ & $2240(17.7)$ & \\
\hline $60-75$ & $32,780(42.5)$ & $10,400(39.2)$ & $16,410(43.2)$ & $5970(47.1)$ & \\
\hline$\geq 75$ & $24,410(31.6)$ & $7280(27.5)$ & $12,660(33.3)$ & $4470(35.2)$ & \\
\hline Sex & & & & & $<0.0001$ \\
\hline Male & $48,570(62.9)$ & $15,480(58.4)$ & $24,490(64.4)$ & $8600(67.8)$ & \\
\hline Female & $28,640(37.1)$ & $11,040(41.6)$ & $13,520(35.6)$ & $4080(32.2)$ & \\
\hline Number of injections, times & $12.0 \pm 2.9$ & $8.7 \pm 3.4$ & $12.3 \pm 1.7$ & $18.1 \pm 4.2$ & $<0.0001$ \\
\hline \multicolumn{6}{|c|}{ Number of injections, categorical } \\
\hline First level $(<10)$ & $26,520(34.4)$ & 26,520 & 0 & 0 & \\
\hline Second level (10-15) & $38,010(49.2)$ & 0 & 38,010 & 0 & \\
\hline Third level $(\geq 15)$ & $12,680(16.4)$ & 0 & 0 & 12,680 & \\
\hline Diabetes & & & & & $<0.0001$ \\
\hline Yes & $27,490(35.6)$ & $10,770(40.6)$ & $12,510(32.9)$ & $4210(33.2)$ & \\
\hline No & $49,720(64.4)$ & $15,750(59.4)$ & $25,500(67.1)$ & $8470(66.8)$ & \\
\hline Hypertension & & & & & $<0.0001$ \\
\hline Yes & $49,900(64.6)$ & $16,940(63.9)$ & $24,420(64.2)$ & $8540(67.3)$ & \\
\hline No & $27,310(35.4)$ & $9580(36.1)$ & $13,590(35.8)$ & $4140(32.7)$ & \\
\hline Hyperlipidemia & & & & & $<0.0001$ \\
\hline Yes & $34,200(44.3)$ & $11,760(44.3)$ & $16,480(43.4)$ & $5960(47.0)$ & \\
\hline No & $43,010(55.7)$ & $14,760(55.7)$ & $21,530(56.6)$ & $6729(53.0)$ & \\
\hline Ischemic heart disease & & & & & $<0.0001$ \\
\hline Yes & $22,990(29.8)$ & $7630(28.8)$ & $11,350(29.9)$ & 4010 (31.6) & \\
\hline No & $54,220(70.2)$ & $18,890(71.2)$ & $26,660(70.1)$ & $8670(68.4)$ & \\
\hline Glaucoma & & & & & $<0.0001$ \\
\hline Yes & $12,730(16.5)$ & $4570(17.2)$ & $5890(15.5)$ & $2270(17.9)$ & \\
\hline No & $64,480(83.5)$ & $21,950(82.8)$ & $32,120(84.5)$ & $10,410(82.1)$ & \\
\hline Follow-up period, years & $3.50 \pm 1.86$ & $3.51 \pm 1.87$ & $3.49 \pm 1.86$ & $3.53 \pm 1.84$ & 0.09 \\
\hline Incident ION & $180(0.23)$ & $40(0.15)$ & $100(0.26)$ & $40(0.32)$ & $<0.01$ \\
\hline
\end{tabular}

$I O N$ ischemic optic neuropathy. Data are presented as mean \pm standard deviation or $\mathrm{n}(\%)$

the risk of ION significantly increased in those who received more anti-VEGF injections, after adjusting for confounders.

AMD is a multifactorial disease, and advanced age is a main predisposing factor. In our study, the study cohort had a mean age of 67.4 years, and nearly one-third of the patients were older than 75 years. These results were compatible with a previous hospital-based study regarding anti-VEGF use among neovascular AMD patients in Taiwan [28]. It is noteworthy that the distribution of comorbidities was higher in our study cohort than in those without AMD in another population-based study in Taiwan [29]. In addition, Table 1 revealed significant differences in age, sex, and comorbidities according to the number of injections. Therefore, the group differences in these variables were adjusted in our subsequent Cox regression analyses.

The Kaplan-Meier curves with the log-rank test (Fig. 1) and Cox regression analyses (Table 2) in our study revealed that a higher number of injections was associated with a higher risk of subsequent ION. Very few case reports have described the onset of ION following the intravitreal injection of anti-VEGF. Hosseini et al. reported a 72-year-old woman with NAION occurring 1 week after the second intravitreal injection of anti-VEGF under the indication of active subfoveal choroidal neovascularization [25]. In their 2009 case report, Ganssauge et al. presented a 51-year-old man with 


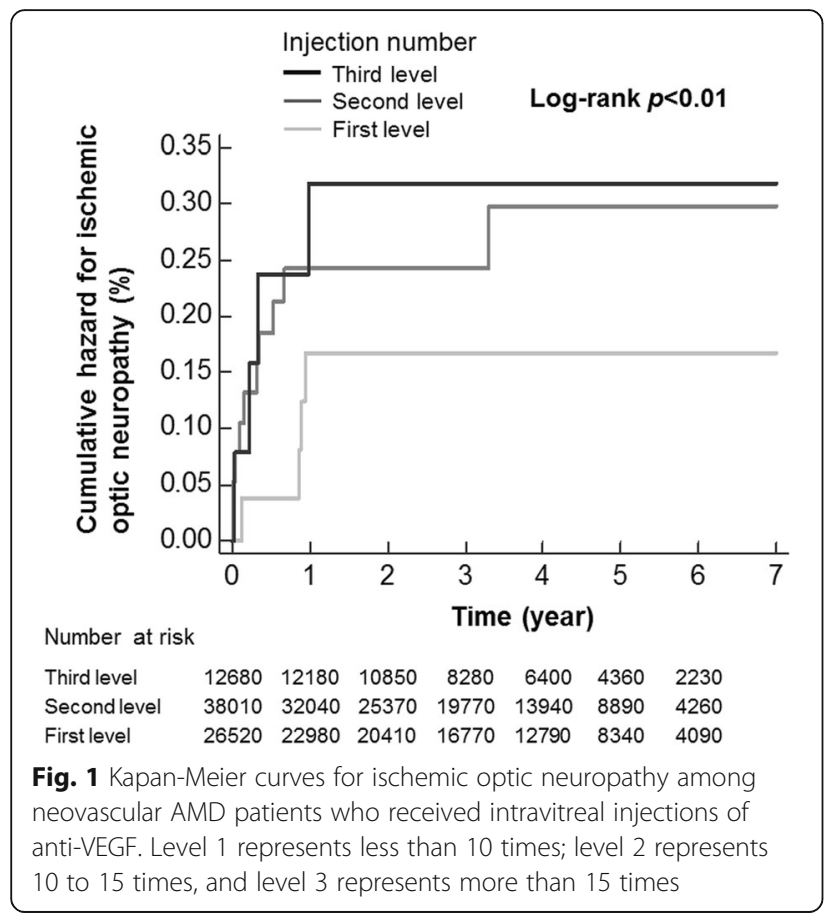

pseudoxanthoma elasticum who received an intravitreal injection for choroidal neovascularization secondary to angioid streaks. Two weeks later, NAION was observed [26]. Huang et al. described a case of a 38-year-old woman with diabetic retinopathy. Three weeks after intravitreal injection of anti-VEGF, anterior ION occurred [27]. Although the elevated IOP that occurs during intravitreal injection might have a compression effect on the optic nerve head, the three patients with ION did not have an elevated IOP. It is possible that the IOP elevation was temporary and was not detected, or it is possible that other pathogeneses, such as anti-VEGF itself, precipitated ION.

Previous studies have shown that VEGF plays a role in modulating both vascular tone and blood flow autoregulation [30]. Ameri et al. found that a sudden decrease in the effective VEGF concentration might be responsible for the closure of normal capillaries [31]. Therefore, anti-VEGF may diminish the blood perfusion to the optic nerve head and cause ION. Additionally, VEGF has been reported to have both neurotrophic and neuroprotective effects [32]. In a diabetic rat model following intravitreal anti-VEGF injection, the rate of apoptosis increased in retinal ganglion cells [33]. It is possible that the optic nerve head is also directly disturbed by antiVEGF and that the influence is additive after repeated injections. Therefore, repeated injections of anti-VEGF may lead to a higher risk of ION.

One limitation of our study is the lack of visual acuity and IOP values in our NHIRD. In addition, we retrieved patients receiving anti-VEGF injections through the procedure codes, but we could not differentiate what kind of anti-VEGF the patients received. These are the inherent drawbacks of our database. Further clinical studies are necessary in order to include these information.

Another limitation of our study is that we lacked controls with similar demographics. More studies regarding the comparison of ION risk between those with antiVEGF and matched controls (without anti-VEGF) are warranted. In our study, patients who underwent fewer than 10 injections (the first-level group) were regarded

Table 2 Risk Factors for ION in the study cohort

\begin{tabular}{|c|c|c|c|c|}
\hline \multirow[t]{2}{*}{ Variables } & \multicolumn{2}{|l|}{ Univariate analysis } & \multicolumn{2}{|l|}{ Multivariate analysis } \\
\hline & Unadjusted HR (95\% Cl) & $p$-value & Adjusted HR (95\% Cl) & $p$-value \\
\hline \multicolumn{5}{|l|}{ Age group, years } \\
\hline$<60$ & Reference & & Reference & \\
\hline $60-75$ & $1.02(0.76-1.39)$ & 0.86 & $1.01(0.75-1.38)$ & 0.91 \\
\hline$\geq 75$ & $1.17(0.86-1.61)$ & 0.31 & $1.09(0.76-1.58)$ & 0.62 \\
\hline Sex (male vs. female) & $0.84(0.65-1.09)$ & 0.19 & $0.82(0.63-1.07)$ & 0.14 \\
\hline \multicolumn{5}{|l|}{ Number of injections } \\
\hline First level $(<10)$ & Reference & & Reference & \\
\hline Second level (10-15) & $1.75(1.21-2.53)$ & $<0.01$ & $1.91(1.32-2.76)$ & $<0.001$ \\
\hline Third level $(\geq 15)$ & $1.99(1.28-3.08)$ & $<0.01$ & $2.20(1.42-3.43)$ & $<0.001$ \\
\hline Diabetes & $1.82(1.36-2.44)$ & $<0.0001$ & $2.03(1.46-2.81)$ & $<0.0001$ \\
\hline Hyspertension & $1.05(0.83-1.35)$ & 0.65 & $1.04(0.85-1.30)$ & 0.65 \\
\hline Hyperlipidemia & $1.27(0.95-1.70)$ & 0.11 & $1.04(0.75-1.43)$ & 0.83 \\
\hline Ischemic heart disease & $1.16(0.85-1.58)$ & 0.35 & $1.20(0.86-1.67)$ & 0.29 \\
\hline Glaucoma & $1.43(1.01-2.03)$ & $<0.05$ & $1.35(0.95-1.92)$ & 0.09 \\
\hline
\end{tabular}

$I O N$ ischemic optic neuropathy, $H R$ hazard ratio, $C l$ confidence interval 
as the reference group. However, the first-level, secondlevel, and third-level groups did not have similar baseline characteristics. Patients in the groups with higher numbers of intravitreal anti-VEGF injections were significantly older and had a significantly higher prevalence in hypertension, hyperlipidemia, and ischemic heart disease. These systemic factors may have affected the incidence of ION. Additionally, older people tend to have more comorbidities including diabetes, hypertension, and ischemic heart disease, which may coexist with ION. These confounding effects potentially complicated our analyses. We tried as possible as we could to adjust these factors in the Cox regression. However, from the perspective of epidemiology, not all confounders were measurable and could be adjusted with statistical methods. Therefore, randomized control trials are still needed to address this issue.

Our study has the strengths of a large sample size, an extended study period, a statistical analysis that adjusted for confounders, and validated diagnoses. In our health care system, the National Health Administration (NHA) frequently checks the cross-consistency of claims and chart data. The NHA also confirmed diagnoses that have been approved by a standard protocol of examinations. Therefore, the diagnoses in our database have a high degree of accuracy. It is noteworthy that in the diagnosis of neovascular AMD, OCT plays an important role. Although the brand or type of OCT machine is not exactly the same in every hospital or clinic, these machines are all useful for detecting the neovasculature and fluid. Some newer modalities, such as spectrum domain OCT, swept source OCT, enhanced depth OCT, and OCT angiography can provide earlier detection of the choroidal neovasculature and can be applied in additional research.

We cannot conclude a causal relationship between repeated anti-VEGF injections and ION. Most likely, the observation that a higher incidence of ION in patients receiving more anti-VEGF injections only reflects a greater need for anti-VEGF in those patients who tend to have ION. At present, all analyses were based on our database, and we derived a positive association between repeated anti-VEGF injections and ION. The underlying mechanism of the association is still not fully understood. Further basic research, animal models, and possibly large-scale prospective cohort studies are needed to unravel the pathogenesis.

\section{Conclusions}

Currently, intravitreal injection of anti-VEGF has become the mainstay of treatment in neovascular AMD. Our study, revealed the possible complication of ION after repeated injections. Our recommendation is not to oppose treatment or change the protocols, which require more time and additional studies. At present, our study is simply a reminder for ophthalmologists to check optic nerve changes following anti-VEGF injections among neovascular AMD patients, especially those who receive a high number of injections. The risk of ION should also be considered when determining the use of anti-VEGF injections for the treatment of neovascular AMD.

\begin{abstract}
Abbreviations
AMD: Age-related macular degeneration; anti-VEGF: Antivascular endothelial growth factor; Cl: Confidence interval; FA: Fluorescein angiography;

HRs: Hazard ratios; ICD-9-CM Codes: International Classification of Diagnoses, Ninth Revision, Clinical Modification Codes; ION: Ischemic optic neuropathy; IOP: Intraocular pressure; NAION: Nonarteritic anterior ION; NHA: National Health Administration; NHIRD: National Health Insurance Research Database; OCT: Optical coherence tomography; SD: Standard deviation; VEGF: Vascular endothelial growth factor
\end{abstract}

\section{Acknowledgements}

Not applicable.

\section{Authors' contributions}

Conceptualization: YYC, PC, YFH, HJC, YCW, CCL, LYH, HHC; Formal analysis: YYC, PC, and HJC; Investigation: YYC, YCW, HHC, and CCL; Methodology: YYC, LYH, and HHC; Validation: YYC, PC, and YFH; Writing the original draft: YYC, and PC. All authors read and approved the manuscript.

\section{Funding}

This research received no specific grant from any funding agency in the public, commercial or not-for-profit sectors.

\section{Availability of data and materials}

The datasets used and/or analysed during the current study are available from the corresponding author on reasonable request.

\section{Ethics approval and consent to participate}

This study was approved by the ethical committee of Yang-Ming University Hospital (2015A018). Permission to collect data was included under ethics approval. The informed consent was exempt according to the Institutional Review Board because each patient record was anonymized and deidentified prior to analysis.

Consent for publication

Not applicable.

\section{Competing interests}

The authors declare that they have no competing interests.

\section{Author details}

${ }^{1}$ Department of Ophthalmology, Taichung Veterans General Hospital, 1650 Taiwan Boulevard Sect. 4, Taichung 40705, Taiwan. ${ }^{2}$ School of Medicine, National Yang-Ming University, Taipei, Taiwan. ${ }^{3}$ Community Medicine Research Center and Institute of Public Health, National Yang-Ming University, Taipei, Taiwan. ${ }^{4}$ School of Medicine, College of Medicine, Fu Jen Catholic University, New Taipei, Taiwan. ${ }^{5}$ Division of Endocrinology and Metabolism, Department of Internal Medicine, Fu Jen Catholic University Hospital, New Taipei, Taiwan. ${ }^{6}$ Department of Medical Education, Fu Jen Catholic University Hospital, New Taipei, Taiwan. ${ }^{7}$ Department of Medical Research, Taichung Veterans General Hospital, Taichung, Taiwan. ${ }^{8}$ Division of Allergy, Immunology, and Rheumatology, Department of Internal Medicine, Taichung Veterans General Hospital, Taichung, Taiwan. ${ }^{9}$ Institute of Biomedical Science and Rong-Hsing Research Center for Translational Medicine, Chung-Hsing University, Taichung, Taiwan. 
Received: 1 March 2019 Accepted: 23 December 2019

Published online: 30 December 2019

\section{References}

1. Iglicki M, Busch C, Zur D, Okada M, Mariussi M, Chhablani JK, Cebeci Z, Fraser-Bell S, Chaikitmongkol V, Couturier A, et al. DEXAMETHASONE IMPLANT FOR DIABETIC MACULAR EDEMA IN NAIVE COMPARED WITH REFRACTORY EYES: The International Retina Group Real-Life 24-Month Multicenter Study. The IRGREL-DEX Study. Retina. 2019;39(1):44-51.

2. Iglicki M, Zur D, Busch C, Okada M, Loewenstein A. Progression of diabetic retinopathy severity after treatment with dexamethasone implant: a 24month cohort study the 'DR-pro-DEX Study'. Acta Diabetol. 2018;55(6):541-

3. Mello Filho P, Andrade G, Maia A, Maia M, Biccas Neto L, Muralha Neto A, Moura Brasil O, Minelli E, Dalloul C, lglicki M. Effectiveness and safety of Intravitreal dexamethasone implant (Ozurdex) in patients with diabetic macular edema: a real-world experience. Ophthalmologica. 2019;241(1):916.

4. Zur D, Iglicki M, Loewenstein A. The role of steroids in the Management of Diabetic Macular Edema. Ophthalmic Res. 2019;62(4):231-6.

5. Iglicki M, Zur D, Fung A, Gabrielle PH, Lupidi M, Santos R, Busch C, Rehak M,

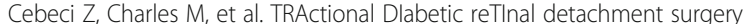
with co-adjuvant intravitreal dexamethasONe implant: the TRADITION STUDY. Acta Diabetol. 2019:56(10):1141-7.

6. Zur D, Iglicki M, Sala-Puigdollers A, Chhablani J. Disorganization of retinal inner layers as a biomarker in patients with diabetic macular oedema treated with dexamethasone implant. Acta Diabetol. 2019. https://doi.org/ 10.1111/aos.14230 [Epub ahead of print].

7. Vedula SS, Krzystolik MG. Antiangiogenic therapy with anti-vascular endothelial growth factor modalities for neovascular age-related macular degeneration. Cochrane Database Syst Rev. 2008;2:Cd005139.

8. Michels S, Rosenfeld PJ, Puliafito CA, Marcus EN, Venkatraman AS. Systemic bevacizumab (Avastin) therapy for neovascular age-related macular degeneration twelve-week results of an uncontrolled open-label clinical study. Ophthalmology. 2005;112(6):1035-47.

9. Bashshur ZF, Haddad ZA, Schakal A, Jaafar RF, Saab M, Noureddin BN. Intravitreal bevacizumab for treatment of neovascular age-related macular degeneration: a one-year prospective study. Am J Ophthalmol. 2008;145(2): 249-56.

10. Rosenfeld PJ, Brown DM, Heier JS, Boyer DS, Kaiser PK, Chung CY, Kim RY. Ranibizumab for neovascular age-related macular degeneration. N Engl J Med. 2006:355(14):1419-31.

11. Hayreh SS. Ischemic optic neuropathies - where are we now? Graefes Arch Clin Exp Ophthalmol. 2013:251(8):1873-84.

12. Biousse V, Newman NJ. Ischemic optic neuropathies. N Engl J Med. 2015; 372(25):2428-36

13. Burde RM. Optic disk risk factors for nonarteritic anterior ischemic optic neuropathy. Am J Ophthalmol. 1993;116(6):759-64.

14. Hayreh SS, Joos KM, Podhajsky PA, Long CR. Systemic diseases associated with nonarteritic anterior ischemic optic neuropathy. Am J Ophthalmol. 1994;118(6):766-80.

15. Jacobson DM, Vierkant RA, Belongia EA. Nonarteritic anterior ischemic optic neuropathy. A case-control study of potential risk factors. Arch Ophthalmol. 1997;115(11):1403-7.

16. Chang YS, Weng SF, Chang C, Wang JJ, Su SB, Huang CC, Wang JY, Jan RL. Risk of Nonarteritic anterior ischemic optic neuropathy following end-stage renal disease. Medicine. 2016;95(12):e3174.

17. Hayreh SS, Zimmerman MB. Nonarteritic anterior ischemic optic neuropathy: clinical characteristics in diabetic patients versus nondiabetic patients. Ophthalmology. 2008;115(10):1818-25.

18. Hayreh SS. Anterior ischemic optic neuropathy. IV. Occurrence after cataract extraction. Arch Ophthalmol. 1980;98(8):1410-6.

19. Hurmeric $V$, Bayer A, Durukan AH, Mutlu FM. Nonarteritic ischemic optic neuropathy developed after capsular block syndrome. Indian J Ophthalmol. 2014;62(3):346-8.

20. McCulley TJ, Lam BL, Feuer WJ. Nonarteritic anterior ischemic optic neuropathy and surgery of the anterior segment: temporal relationship analysis. Am J Ophthalmol. 2003;136(6):1171-2.

21. Choudhari NS, George R, Kankaria V, Sunil GT. Anterior ischemic optic neuropathy precipitated by acute primary angle closure. Indian J Ophthalmol. 2010;58(5):437-40.
22. Kuriyan $A E$, Lam BL. Non-arteritic anterior ischemic optic neuropathy secondary to acute primary-angle closure. Clin Ophthalmol. 2013;7:1233-8.

23. Kim KN, Kim CS, Lee SB, Lee YH. Delayed non-arteritic anterior ischemic optic neuropathy following acute primary angle closure. Korean J Ophthalmol. 2015;29(3):209-11.

24. Falavarjani KG, Nguyen QD. Adverse events and complications associated with intravitreal injection of anti-VEGF agents: a review of literature. Eye (Lond). 2013;27(7):787-94.

25. Hosseini H, Razeghinejad MR. Anterior ischemic optic neuropathy after intravitreal injection of bevacizumab. J Neuroophthalmol. 2009;29(2):160-1.

26. Ganssauge M, Wilhelm H, Bartz-Schmidt KU, Aisenbrey S. Non-arteritic anterior ischemic optic neuropathy (NA-AION) after intravitreal injection of bevacizumab (Avastin) for treatment of angoid streaks in pseudoxanthoma elasticum. Graefes Arch Clin Exp Ophthalmol. 2009;247(12):1707-10.

27. Huang JY, Ozaki H, Hayashi H, Uchio E. Anterior ischemic optic neuropathy following intravitreal bevacizumab. Jpn J Ophthalmol. 2010;54(3):252-4.

28. Chang YS, Lee WJ, Lim CC, Wang SH, Hsu SM, Chen YC, Cheng CY, Teng YT, Huang $\mathrm{YH}$, Lai CC, et al. Real-world use of ranibizumab for neovascular agerelated macular degeneration in Taiwan. Sci Rep. 2018;8(1):7486.

29. Tsai DC, Chen SJ, Huang CC, Yuan MK, Leu HB. Age-Related Macular Degeneration and Risk of Degenerative Dementia among the Elderly in Taiwan: A Population-Based Cohort Study. Ophthalmology. 2015;122(11): 2327-2335.e2322.

30. Hayreh SS. Blood flow in the optic nerve head and factors that may influence it. Prog Retin Eye Res. 2001;20(5):595-624.

31. Ameri H, Chader GJ, Kim JG, Sadda SR, Rao NA, Humayun MS. The effects of intravitreous bevacizumab on retinal neovascular membrane and normal capillaries in rabbits. Invest Ophthalmol Vis Sci. 2007:48(12):5708-15.

32. Nicoletti JN, Shah SK, McCloskey DP, Goodman JH, Elkady A, Atassi H, Hylton D, Rudge JS, Scharfman HE, Croll SD. Vascular endothelial growth factor is up-regulated after status epilepticus and protects against seizureinduced neuronal loss in hippocampus. Neuroscience. 2008;151(1):232-41.

33. Park HY, Kim JH, Park CK. Neuronal cell death in the inner retina and the influence of vascular endothelial growth factor inhibition in a diabetic rat model. Am J Pathol. 2014;184(6):1752-62.

\section{Publisher's Note}

Springer Nature remains neutral with regard to jurisdictional claims in published maps and institutional affiliations.

Ready to submit your research? Choose BMC and benefit from:

- fast, convenient online submission

- thorough peer review by experienced researchers in your field

- rapid publication on acceptance

- support for research data, including large and complex data types

- gold Open Access which fosters wider collaboration and increased citations

- maximum visibility for your research: over $100 \mathrm{M}$ website views per year

At $\mathrm{BMC}$, research is always in progress.

Learn more biomedcentral.com/submission 